\title{
¿Cómo investigar un problema que involucra a distintas disciplinas? (propuestas para la investigación en bioética)
}

\author{
Cecilia Pourrieux ${ }^{1}$
}

\section{Introducción}

Numerosas son las transformaciones que ha experimentado el conocimiento en un sentido general, desde la clásica división griega del saber entre teórico y práctico. Los avances científicos -especialmente en el plano de las ciencias de la vida- se presentan a la reflexión en forma vertiginosa con problemas inéditos sobre los cuales es necesario debatir. La dinámica entre soluciones y problemas se ha exacerbado. Lo que se presenta en un momento como respuesta a un conflicto, genera en lo inmediato la aparición simultánea de nuevos problemas, distintos y urgentes.

Caracterizar el desarrollo de la ciencia para comprender el momento actual es algo que excede el objetivo de este trabajo. Nuestra meta se dirige a indagar quien debe abordar la investigación de algún campo del saber actual, cualquiera sea este, que a su vez es atravesado por otros tantos campos disciplinares. En este sentido, la integración y el debate entre diferentes tipos de saber son el sello distintivo de los tiempos actuales. La pregunta que se le plantea al investigador es ¿cómo investigar un problema que involucra a distintas disciplinas? Proponemos abordar este interrogante tomando a la investigación en bioética como paradigma investigativo. Esta elección responde al hecho de que ella misma es una disciplina relativamente nueva en la cual convergen distintos saberes. En sus debates intervienen no sólo perspectivas legales, científicas, sociales, morales, políticas sino otras tantas más. La interrelación entre ellas implica procesos complejos en la toma de decisiones respecto a nuevos conflictos, en donde se cuestionan normas y valores socialmente legitimados. Este diálogo entre múltiples disciplinas, involucra diferentes aspectos y en función de ellos es que vamos a mostrar dos posibles enfoques para analizar el problema:

1 Cecilia Pourrieux es egresada, docente e investigadora de la Universidad Nacional de Tucumán hasta el año 2008. Actualmente se desempeña como docente e investigadora en la Universidad Nacional de Lanús, en el Área Ética. Diplomada en estudios superiores en bioética, FLACSO, 2013. Último trabajo publicado: "Reproducción asistida: una mirada desde la bioética" del libro: Responsabilidad Profesional de los Médicos. Ética, Bioética y Jurídica. Civil y Penal. Tomo Il. Coordinador Dr. Oscar Ernesto Garay. Editorial, Edit. La Ley, Bs As, 2014. 
1) Epistemológico.

2) Histórico.

Ambos enfoques son complementarios y se presentan interconectados en la investigación. El abordaje histórico permite ir reconstruyendo el objeto de análisis para delimitar su ubicación en el plano epistemológico, en donde encontraremos una caracterización de la ciencia, alejada de una concepción aséptica o neutral del conocimiento. En las conclusiones se expondrá el carácter práctico y contextual de las investigaciones y la necesidad de introducir el debate entre las diferentes disciplinas.

\section{¿Disciplinar, interdisciplinar, transdisciplinar o multidisciplinar?}

De la modernidad a la actualidad, hay un proceso de especialización cada vez mayor que bien puede ser ejemplificado con la afirmación cartesiana de: "dividir cada una de las dificultades que examinare en tantas partes como fuese posible y en cuantas requiriese su mejor solución"(Descartes, 1980). Esta regla es un claro ejemplo del inicio de un proceso de división del conocimiento, que está a la base de toda disciplina y del problema de la interdisciplinariedad, (Peñuela Velásquez, 2005). Esto supone el siguiente obstáculo: a medida que se profundiza en el desarrollo y la comprensión de cada una de las partes, se aleja cada vez más de la comprensión del fenómeno como un todo. Este pareciera que fue el desarrollo del saber científico, ligado al concepto de disciplina, en donde paulatinamente, fue considerada como sinónimo de especialización. El debate de estos últimos años considera que esta fragmentación debe ser superada, para integrar todo aquello que una vez fue separado, pasando de lo disciplinar a lo interdisciplinario.

La cuestión central es la siguiente: ¿cómo se integran los distintos saberes frente a un problema inédito que se eleva sobre los pilares de aquellos campos clásicos del saber? Es así que surge el debate en torno a como se define la intersección de estos nuevos saberes: ¿son interdisciplinarios, transdisciplinares o multidisciplinares? Claramente la pregunta se origina en el terreno de la praxis y para ilustrarlo apelaremos a las distinciones que realiza Avenai Garrafa (2005) desde el ámbito de la bioética. Destaca en primer lugar que el error más frecuente es confundir suma con integración de disciplinas. Señala como primera medida que conviene distinguir la modalidad que puede tener en cada caso, esta integración. Un común denominador a la distinción entre multidisciplinariedad, interdisciplinariedad y transdisciplinariedad, es que todas tienden a rebasar los límites tradicionales de una sola disciplina, pero su delimitación parte claramente de objetivos distintos. Cuando un problema que tradicionalmente era abordado por una disciplina, trasciende los límites de ella y es analizado desde otras perspectivas, estamos en el terreno de la pluridisciplinariedad o-según su denominación actual- multidisciplinariedad. En este modo de integración, en lo que concierne a la bioética, el debate se enriquece con diferentes perspectivas, sin dejar de pertenecer al terreno médico. Un claro ejemplo de ello, puede ser la eutanasia, que en los debates bioéticos escucha las voces del derecho, la ética y no solo de la medicina. Pero, cuando el método de una disciplina se transfiere a otras tantas, estamos en el terreno de la interdisciplinariedad. Siempre en el terreno de la práctica, existen grados de aplicación. Garrafa ilustra estos grados de la siguiente manera: 
Cecilia Pourrieux / ¿Cómo investigar un problema que involucra a [...]/129

Por ejemplo, los métodos de la ingeniería genética (biología) transferidos a la medicina o al derecho, llevan al surgimiento de nuevas referencias con relación a las comprobaciones de la paternidad. Un segundo "grado epistemológico": la transferencia de métodos de la lógica dialéctica para la salud pública produce análisis diferenciados en la epistemología del derecho (justicia social). Y un tercer "grado de generación de nuevas disciplinas", (Garrafa, 2005, p.70) cuando varios conocimientos se juntan para crear otra disciplina, cuyo ejemplo está como que cortado al talle para la bioética.

La transdisciplinariedad, siguiendo al mismo autor, tiene como finalidad la unidad del conocimiento, esto es, comprender la realidad en su totalidad. De ahí su distinción con las dos modalidades anteriores, que no trascienden el ámbito disciplinar, a diferencia de ésta última que tiene como pretensión, de acuerdo al prefijo "trans", ir 'más allá de este campo, atravesar lo disciplinar, ubicarse 'entre ellas'. Es por esto mismo que muchos autores consideran que este modelo, es el adecuado para caracterizar la actividad de la bioética en la medida que trascendiendo los distintos saberes, sin reducir sus problemas a una sola disciplina, habita entre los intersticios conceptuales problematizando las distintas respuestas que brinda cada saber. Esta modalidad quizás sea difícil de concebir para una mentalidad forjada en los parámetros modernos en donde cada objeto está determinado por un campo del saber. Pero aquello que aparece difícil en el terreno de la especulación, en el ámbito de la praxis, aparece en forma necesaria y urgente. El mejor ejemplo de ello es lo que acontece en la bioética. En donde, antes que disputarse un objeto de conocimiento, el problema desborda los límites de lo conceptual. Es concreto. Necesita hundir sus raíces en la realidad porque en su resolución no están comprometidas categorías especulativas sino vidas humanas, la salud y la enfermedad, la posibilidad o no de una cura. Y en este terreno, urgen las soluciones. Esta necesidad, no acontece sólo en el ámbito de la bioética, sino en cada debate que pone en juego un curso de acción. Es por esto que para examinar estas cuestiones, se hace necesario examinar la historia y el contexto en el cual aparecen los problemas y se plantean las soluciones.

\section{De los principios al contexto}

Todo lo que mueve a los hombres tiene que pasar necesariamente por sus cabezas; pero la forma que adopte dentro de ellas depende en mucho de las circunstancias.

(Engel, 1975, p. 55)

La especie humana, desde siempre, se abocó a la tarea de comprender todo lo que existe. Pero no al modo de una pregunta abstracta, sino como una forma de resolver problemas concretos que le permitieran sobrevivir en este mundo. Numerosas son las teorías epistemológicas que giran en torno al debate de la dinámica actual del conocimiento. Un ejemplo de ello, es Barrow que en su libro Teoría del todo intenta explicitar el alcance y las posibilidades de encontrar una ley que pueda dar detalle del origen y la evolución, no sólo de este mundo, sino del universo todo.(Rieznik, 2005) El reduccionismo, por el contrario, se ubica en las antípodas de esta perspectiva y consiste en el intento de analizar las partes de un fenómeno determinado con la intención posterior de repro- 


\section{0/ Perspectivas Metodológicas /19/Vol. II /Año 2017}

ducir el conjunto. Las posiciones críticas al reduccionismo, señalan que el conocimiento científico no avanzó de esta manera, sino por el contrario, la historia nos demuestra que su desarrollo fue a través de la acción vinculante e integradora entre las diferentes áreas del saber. El esfuerzo metodológico apunta a investigar no sólo a cada elemento sino también los vasos comunicantes que existen entre ellos para poder abordar la realidad como una totalidad orgánica, (Rieznik, 2005). En el apartado anterior, además vimos que por el desarrollo mismo de los problemas, es necesario superar esta fragmentación del conocimiento. Los conflictos actuales, así lo demandan, dado que asistimos a un desarrollo como nunca antes se vio, de las capacidades humanas en su intento por entender y transformar la naturaleza. Y en ese esfuerzo los problemas con los que se encuentra el investigador son reales. En este apartado intentaremos demostrar la integración en una totalidad, del avance tecno-científico con el contexto social, económico, cultural y político. Lo primero en señalar será el carácter histórico de estos avances. En otras palabras, son construcciones humanas que acontecen a lo largo del tiempo. De ahí la necesidad de aproximarnos a ellas en términos de proceso, o sea como una materia sujeta a desarrollo histórico. Esta es justamente la base del materialismo que en la delimitación con el idealismo considera que la historia se desenvuelve en distintas fases sucesivas legitimadas en una época determinada y que, con el transcurso del devenir, indefectiblemente y por la misma dinámica de lo real, será reemplazada por formas nuevas:

[...] la gran idea cardinal de que el mundo no puede concebirse como un conjunto de objetos terminados sino como un conjunto de procesos en el que las cosas parecen estables, al igual que sus reflejos mentales en nuestras cabezas, los conceptos, pasan por un cambio ininterrumpido, por un proceso de devenir y desaparecer (Rieznik, 2005, p. 138).

Este punto de vista, cabe aclarar, no implica un determinismo en el cual el hombre es una hoja al viento llevada por fuerzas ajenas a él, ni una concepción mecanicista en la cual todo se resuelve en base al factor económico, puesto que la dinámica del conocimiento solo puede ser entendida poniendo en el centro a la acción humana, como motor y por lo tanto al hombre como agente de la historia.

Otro factor a tener en cuenta es que las investigaciones bio-tecno-científicas no se desenvuelven en un escenario ideal, sino en grupos sociales en los cuales conviven múltiples variables políticas y culturales, que además están atravesados por diferentes intereses económicos. Esto último merece una observación más detenida dado que es un problema que reviste distintos aspectos. En un sistema político y social en el cual las regulaciones parecieran regirse en base al valor del mercado ¿el conocimiento sale indemne de las generales de esta ley? Si bien desde hace ya tiempo que la neutralidad del conocimiento científico ha sido legítimamente cuestionada, importa ver qué papel desarrolla el investigador, en este marco en el cual conviven laboratorios e intereses empresariales, investigación y mercado. De ahí, la necesidad de contextualizar cada debate. En ese sentido y retomando como ejemplo a la bioética, vemos que ésta tiene la característica de introducir elementos polémicos que exigen ser debatidos en la sociedad.

Veamos brevemente los criterios con los que estos debates se desenvolvieron en la historia de esta disciplina. Por ejemplo: la teoría de los principios, en tanto fundamentación 
Cecilia Pourrieux / ¿Cómo investigar un problema que involucra a [...]/131 moral para la bioética, fue enunciada en 1979 por Tom Beauchamp y James Childress en su libro Principios de Ética Biomédica. Su predecesor fue el Informe Belmont producido por la Comisión Nacional para la Protección de Sujetos Humanos en la Investigación Biomédica $y$ de la Conducta, creada por el Congreso de los Estados Unidos en 1974. El objetivo era identificar los principios éticos básicos subyacentes en la conducta de las investigaciones y servir para desarrollar pautas y regulaciones administrativas (U.S.Congress, 1978). Esa primera comisión nacional de bioética fue propuesta en 1973 ante la fuerte tensión política de los defensores de los derechos civiles que reclamaban en torno a investigaciones realizadas en ese momento. En el libro de Beauchamp y Childress los principios serían cuatro: beneficencia, no maleficencia, autonomía y justicia. (Tealdi, 2005) Esta teoría de los principios, desempeñó un rol central en cada uno de los debates desarrollados en el seno de la bioética. Casi dos décadas después, estalla el escándalo por las investigaciones para reducir la transmisión perinatal del HIV, en donde se denuncia el tratamiento con placebo (aunque ya existía tratamiento para el HIV) de un grupo de control en África y República Dominicana. Los patrocinantes eran institutos de Estados Unidos, los cuales argumentaban en su defensa que "el no tratamiento" era el estándar local (Tealdi, 2005).

Desafortunadamente, ejemplos recientes como este abundan. La polémica que se desarrolla actualmente en torno a la ética de la investigación tiene este eje: la industria farmacológica, utiliza las investigaciones multicéntricas, en distintos países de América latina en especial, en las cuales busca la manera de acrecentar sus ganancias investigando en un marco regulatorio que les permite evadir la discusión en torno a los derechos humanos básicos. Vulnerando no solo los derechos de los sujetos de investigación sino de la accesibilidad de la población toda a esos medicamentos.

Tomando como ejemplo el debate en la bioética, la primera conclusión a la que arribamos es la siguiente: el debate en torno a la prevalencia de unos principios sobre otros, tal como ocurrió en la ética de la investigación, nos aleja de la realidad. Mientras tanto, los hechos son más que elocuentes: sujetos de investigación "usados" en países pobres para medicamentos que serán utilizados en países ricos; muertes maternas en ascenso por la práctica de aborto clandestino; uso privado con fines de comercialización en la investigación de distintas terapias génicas. En fin, los ejemplos abundan. Si acordamos que el objetivo de la investigación es el de no perder de vista los hechos y al mismo tiempo ubicarse en la posición de agente de los acontecimientos, urge entonces, buscar algunos criterios que contengan en sí la potencialidad de contextualizar el debate.

\section{Reflexiones finales}

Cuando se habla de contextualizar los problemas, se considera que es de la más urgente necesidad realizar un análisis en base a datos que provengan de la realidad social en cuestión. Para ilustrar esto, tomemos el caso en bioética de la polémica en torno a la política sanitaria aplicada a la salud reproductiva por un lado y la legalización del aborto por el otro. Estos problemas necesitan ser debatidos, por ejemplo, a la luz de las estadísticas de muerte materna, de embarazos adolescentes, etc. Estas variables son reales y permiten dirigir la polémica en base a datos concretos. De acuerdo al apartado anterior, podemos extraer varias conclusiones. Tomando en cuenta el dinamismo inherente a lo 


\section{2/ Perspectivas Metodológicas /19/Vol. II /Año 2017}

real, las investigaciones, deben redefinirse constantemente no solo en lo que respecta a sus problemas, sino también en cuanto a su método y los obstáculos que debe sortear. Por ejemplo, conviene revisar cómo va transformándose el objetivo de las investigaciones tecno-científicas a la luz de la aparición de los nuevos productos y las modificaciones que en ellas se suceden a raíz de su comercialización. Puede afirmarse que hasta finales del siglo XIX existe todavía la figura del investigador que, inmerso en su laboratorio, arriba a conocimientos que le permiten avanzar sobre un tema específico. Esta relación entre sujeto-investigador-estado es justamente la que sufrirá los principales cambios a comienzos del siglo XX. Tomemos como ejemplo las transformaciones de los objetivos de la industria farmacológica a medida que comienzan a insertarse los nuevos medicamentos en el mercado. Así, el estado por un lado y las empresas privadas por el otro, verán en estos avances, la posibilidad estratégica de aplicarlos con fines comerciales. Para un estado, pasa a ser una cuestión política importante en la medida en que aparece una nueva posibilidad de progreso económico. EEUU es un claro ejemplo de ello. Otro obstáculo frecuente a la hora de investigar es el de confundir neutralidad valorativa de las ciencias con objetividad científica. En el primer caso y tomando el contexto en el que estas se desenvuelven, sobran argumentos para cuestionarlas. Sin embargo, se puede considerar la objetividad científica en tanto actividad social "con las limitaciones del momento histórico que manifiesta. Su contenido está ligado a la capacidad del hombre de comprender los procesos de los cuales forma parte y en los cuales interviene". (Rieznik, 2005) En base a esto, afirmamos que el escenario en el cual se desenvuelven las investigaciones científicas, no es el de un laboratorio aséptico. Un investigador, para no caer en una desvío distraccionista, debe incorporar en su análisis todos los elementos que permitan comprender que los debates actuales se desarrollan en un marco social y político atravesado de fuerzas antagónicas en constante tensión. Incorporando a los mismos la existencia de intereses económicos y políticos que podrían llevar a la distorsión de cualquier debate. Finalmente, apelaremos a la bioética para mostrar a modo de ejemplo, el vínculo existente entre los problemas que hunden sus raíces en la realidad, una disciplina (que puede ser cualquiera) y los objetivos que debe plantearse todo investigador:

No es casual que la bioética haya surgido en momentos en que la racionalidad, la ciencia, los estados, la democracia y la economía no cumplieron sus promesas. Las de que iba a haber salud para todos en el año 2000 por ejemplo, o las de constituir organismos internacionales que aseguraran la paz, o las de garantizar la alimentación a todos los hombres o poner al alcance de todo el que quisiera los instrumentos para el conocimiento y la creación. La tarea de la bioética es la de no olvidar esos compromisos que la humanidad tiene con sus componentes y recordarlos en todas las circunstancias en que estén en peligro, en todas las circunstancias en que la vida siga siendo el precio de la justicia. (Pfeiffer, 2002)

Para concluir, el conocimiento por el conocimiento mismo no debería ser concebido como una meta en la investigación. Más bien constituye un obstáculo que desvía la conciencia. Por el contrario, desde este espacio proponemos que cada investigador oriente su producción en base a un compromiso con la humanidad, no en un sentido abstracto, sino encarnada en la figura de cada hombre. 
Cecilia Pourrieux / ¿Cómo investigar un problema que involucra a [...]/133

\section{Bibliografía}

— Descartes, R., "Discurso del Método" en Obras escogidas. Editorial Charcas, Bs As, 1980.

- Peñuela Velásquez, A,: "La transdisciplinariedad. Más allá de los conceptos, la dialéctica" en Andamios v. 1 no 2, México, 2005. http://www.scielo.org.mx/scielo. php?pid=S1870-00632005000300003\&script=sci_arttext\#notas

- Garrafa, V.: "Multi-inter-transdisciplinariedad, complejidad y totalidad concreta en bioética" en: Estatuto epistemológico de la bioética. Garrafa, Kotow y Saada. UNAM, México, 2005.

- Engels F., Plejánov G.: Ludwig Feuerbach y el fin de la filosofia clásica alemana. P. 55. Ed. Siglo XXI, Cuadernos del Pasado y el Presente, 1975.

- Rieznik, P: El mundo no empezó en el 4004 antes de Cristo. Marx, Darwin y la ciencia moderna. (Prefacio) Editorial Biblos, Buenos Aires, 2005.

— Tealdi, J.C.: "Los principios de Georgetown" en V.Garrafa, A.Saada, M.Kottow (coords), Estatuto Epistemológico de la Bioética, México, Universidad Nacional Autónoma de México-UNESCO, 2005, p.35-54.

- Tealdi, J.C. "Etica de la investigación: el principio y el fin de la bioética". Summa Bioética.-Organo de la Comisión Nacional de Bioética-, México, Año I, Número Especial, Septiembre de 2003, p.69-72.

- Pfeiffer; M. L., "El ser humano como objeto. Ciencia y ética”. Revista Jurídica de Buenos Aires. Bioética y Derechos Humanos, Buenos Aires, 2006.

— Pfeiffer, M. L., "Bioética ¿para qué? En Cuadernos de Ética No 30 Buenos Aires, 2002.

— Jungues, R., "El Estatuto espitemológico de la bioética" http://www.unesco.org.uy/ shs/fileadmin/templates/shs/archivos/TrabajosLibres-Bioetica/16.\%20El\%20estatuto\%20espistemologico.pdf 